\title{
Efficacy of anastrozole in the treatment of hypogonadal, subfertile men with body mass index $\geq 25 \mathrm{~kg} / \mathrm{m}^{2}$
}

\author{
Tejash Shah ${ }^{1}$, Themba Nyirenda ${ }^{2}$, David Shin ${ }^{3,4} \wedge$ \\ ${ }^{1}$ Division of Urology, Rutgers New Jersey Medical School, Newark, NJ 07103, USA; ${ }^{2}$ Department of Research, Hackensack University Medical \\ Center, Hackensack, NJ 07601, USA; ${ }^{3}$ Department of Urology, Hackensack University Medical Center, Hackensack, NJ 07601, USA; ${ }^{4}$ Department \\ of Urology, Hackensack Meridian School of Medicine, Nutley, NJ 07110, USA \\ Contributions: (I) Conception and design: All authors; (II) Administrative Support: D Shin; (III) Provision of study materials or patients: D Shin; (IV) \\ Collection and assembly of data: T Shah, D Shin; (V) Data analysis and interpretation; All authors; (VI) Manuscript writing: All authors; (VII) Final \\ approval of manuscript: All authors. \\ Correspondence to: David Shin, MD. Department of Urology, HackensackUMC, 360 Essex St., Suite 403, Hackensack, NJ 07601 , USA. \\ Email: David.Shin@hmhn.org.
}

Background: Anastrozole is a non-steroidal fourth generation aromatase inhibitor that stops the conversion of testosterone to estradiol and has been used as empiric medical therapy for the treatment of male infertility in men with an abnormal testosterone-to-estradiol ratio $<10$ in order to increase endogenous testosterone levels. This study sought to evaluate the efficacy of anastrozole in the treatment of hypogonadal, subfertile men with body mass index greater than $25 \mathrm{mg} / \mathrm{kg}^{2}$ with respect to hormonal profile, semen parameters and overall fertility status.

Methods: Retrospective chart review was performed of hypogonadal, subfertile men with body mass index $\geq 25 \mathrm{~kg} / \mathrm{m}^{2}$ who were treated with anastrozole ( $1 \mathrm{mg}$ daily). Hormonal measurements and semen analysis prior to and after treatment was analyzed in 30 men. Total motile count was calculated from semen analysis. Clinical pregnancy rates were recorded.

Results: Men treated with anastrozole had increases in follicle stimulating hormone (4.8 versus 7.6 IU/L, $\mathrm{P}<0.0001$ ), luteinizing hormone (3.4 versus $5.4 \mathrm{IU} / \mathrm{L}, \mathrm{P}<0.0001$ ), testosterone (270.6 versus $412 \mathrm{ng} / \mathrm{dL}$, $\mathrm{P}<0.0001$ ) and testosterone-to-estradiol ratio (9 versus 26.5, $\mathrm{P}<0.0001)$ and decrease in estradiol level (32 versus $15.9 \mathrm{pg} / \mathrm{mL}, \mathrm{P}<0.01)$ after 5 months of therapy. Increases in sperm concentration $(7.8$ versus $14.2 \mathrm{million} / \mathrm{mL}$, $\mathrm{P}<0.001)$, total motile count (12.6 versus 17.7 million, $\mathrm{P}<0.01)$ and strict morphology (3.0\% versus $3.5 \%$, $\mathrm{P}<0.05$ ) was appreciated. Clinical pregnancy rate for our cohort was 46.6\% (14 of 30), with 71.4\% (10 of 14 ) conceiving through in vitro fertilization, $14.2 \%$ (2 of 14) through intrauterine insemination and $14.2 \%(2$ of 14$)$ through natural intercourse.

Conclusions: Anastrozole improves hormonal profiles and semen parameters in hypogonadal, subfertile men with body mass index over $25 \mathrm{~kg} / \mathrm{m}^{2}$ and may aid in achieving pregnancy especially in conjunction with assisted reproductive techniques.

Keywords: Anastrozole; aromatase inhibitor; assisted reproductive technique; male infertility; obesity

Submitted May 11, 2020. Accepted for publication Sep 25, 2020.

doi: 10.21037/tau-20-919

View this article at: http://dx.doi.org/10.21037/tau-20-919

\footnotetext{
$\wedge$ ORCID: 0000-0002-0945-7029.
} 


\section{Introduction}

Approximately $15 \%$ of couples have difficulty achieving pregnancy after one year of unprotected intercourse, with impaired sperm quality or production contributing as a male factor in $30 \%$ to $50 \%$ of all infertile couples (1-3). Thirty to $70 \%$ of men with male infertility have some degree of endocrine dysfunction $(4,5)$ with $43 \%$ prevalence of hypogonadism observed in men with oligospermia (6). Because spermatogenesis is dependent upon on high levels of intratesticular testosterone (7), empiric medical therapy for male infertility such as aromatase inhibitors has been used to increase endogenous testosterone levels in order to improve spermatogenesis (3).

Aromatase is a cytochrome P-450 enzyme that converts testosterone to estradiol and androstenedione to estrone, respectively. It is found in female reproductive tissue, adipose tissue, testes, liver and brain (8). Aromatase inhibitors suppress the peripheral metabolism of testosterone to estradiol which in turn leads to increased testosterone levels and decreased estrogen levels. The combined effect of increasing endogenous testosterone helps to further stimulate spermatogenesis while lower estrogen levels helps to minimize its inhibitory effect on the hypothalamic-pituitary-gonadal (HPG) axis.

Anastrozole is a nonsteroidal aromatase inhibitor that causes reversible enzyme inhibition and has been shown to improve hormonal profile and semen parameters in male factor infertility patients (8-10). However, its effect in overweight or obese, subfertile men is unknown. Obesity has been postulated to contribute to endocrine dysfunction in male factor infertility in a variety of ways, most notably estrogen excess from the peripheral aromatization of testosterone to estrogen in adipose tissue leading to a direct inhibitory effect on the HPG axis with subsequent impairment of spermatogenesis (11). A systematic review conducted by Campbell et al. demonstrated that obese men were more likely to experience infertility, decreased rates of live births associated with assisted reproductive technology (ART) and increased risk of pregnancy non-viability (12). In addition, obese men have been shown to have increased percentage of sperm with low mitochondrial membrane potential, DNA fragmentation, and abnormal morphology further affecting fertility potential (13).

The purpose of our study was to examine the responses in hormonal profile, semen parameters and overall fertility status over time following the initiation of anastrozole in subfertile men with body mass index (BMI) greater than $25 \mathrm{~kg} / \mathrm{m}^{2}$. We present the following article in accordance with the STROBE reporting checklist (available at http://dx.doi.org/10.21037/ tau-20-919).

\section{Methods}

All patients presented to 1 author (DS). Hypogonadal, subfertile men with $\mathrm{BMI} \geq 25 \mathrm{~kg} / \mathrm{m}^{2}$ who were treated with anastrozole ( $1 \mathrm{mg}$ daily) between 2008 and 2014 were included in this study as BMI $\geq 25 \mathrm{~kg} / \mathrm{m}^{2}$ is considered overweight. Indication for anastrozole therapy included testosterone level $<300 \mathrm{ng} / \mathrm{dL}$ or bioavailable testosterone $<155 \mathrm{ng} / \mathrm{dL}$. Exclusion criteria were men with past exogenous testosterone use or with abnormal results on karyotype analysis, Y chromosome microdeletion or cystic fibrosis genetic analysis.

Data on demographics (age, BMI), medical history, genitourinary physical examination, adverse events, serum hormonal evaluation, liver function test (LFT), complete blood count (CBC) and semen analyses were collected prior to and after initiation of treatment. Hormonal concentrations, LFT and CBC levels were obtained before treatment and at 1 month, 3 month and 5 months after prescribing anastrozole. Hormonal analysis was attempted to be obtained between 7:00 am and 11:00 am by venipuncture. Hormonal evaluation included assessment of serum follicle stimulating hormone (FSH), luteinizing hormone ( $\mathrm{LH})$, testosterone $(\mathrm{T})$, estradiol (E), albumin, sex hormone binding globulin (SHBG) and prolactin. Commercially available laboratories were used for the hormonal testing and testosterone was measured via chemiluminescent immunoassays. Only patients with complete hormonal profile and semen analysis data for the study time points were included.

Semen analysis were performed before treatment and at 5 months after treatment. Semen samples were collected after an abstinence period of $2-5$ days and processed within 1 hour of ejaculation. Semen analysis parameters that were analyzed included volume of ejaculate $(\mathrm{mL})$, sperm concentration (million per $\mathrm{mL}$ ), motility (percent) and strict morphology (percent).

\section{Statistical analysis}

Data from evaluable patients were presented using descriptive statistics as follows: continuous variables were summarized as mean (standard deviation) when the data were normally distributed and expressed as median (interquartile range: 25 th- 75 th percentile) for non- 
Table 1 Baseline demographics of study population treated with anastrozole

\begin{tabular}{ll}
\hline Demographic & Value \\
\hline Age (years), median (IQR) (range) & $34.0(33.0-38.0)(26.0-62.0)$ \\
Partner age (years), mean (SD) (range) & $33.0(3.7)(26.0-42.0)$ \\
Weight (Ibs), median (IQR) (range) & $202.5(180.0-235.0)(155.0-400.0)$ \\
Height (in), mean (SD) (range) & $70.0(3.7)(60.0-80.0)$ \\
BMI (kg/m²), median (IQR) (range) & $28.7(26.6-32.2)(25.1-54.2)$ \\
Time since infertility diagnosis (years), median (IQR) (range) & $1.0(1.0-3.0)(0.3-7.0)$ \\
Smoking status, yes/no & $3 / 25$ \\
Left varicocele (Grade 1 or higher), yes/no & $8 / 22$ \\
Right varicocele (Grade 2 or higher), yes/no & $2 / 28$ \\
Primary infertility, yes/no & $27 / 3$ (secondary infertility) \\
\hline
\end{tabular}

Lbs, pounds; In, inches; IQR, interquartile range; SD, standard deviation.

normally distributed data. The assumption of normality was assessed using Shapiro-Wilk test. Categorical variables were presented as frequency (percentage). Comparison of continuous variables between independent groups was conducted using independent sample two-sided t-test or Wilcoxon rank sum test, as appropriate. We tested whether there was a difference in FSH, LH, testosterone and estradiol at baseline compared to levels reported at 1 , 3 and 5 -month follow-up visits using a two-sided $t$-test or Wilcoxon signed-rank, sign test, as appropriate. Comparison of categorical variables was performed using Fisher's exact test or Pearson's chi-square test, as appropriate. Any $\mathrm{P}<0.05$ was considered statistically significant. All data analysis was performed using the statistical software SAS 9.4 (SAS Institute Inc., Cary, North Carolina, USA).

The study was conformed to the provisions of the Declaration of Helsinki (as revised in 2013) and approved by the Ethics Committee of Hackensack University Medical Center/Institutional Review Board (IRB number: Pro00006190). Because of the retrospective nature of the research, the requirement for informed consent was waived.

\section{Results}

Out of 53 total men, 30 subfertile men who met inclusion criteria were treated with anastrozole with follow-up hormonal analyses performed at 1,3 , and 5 months and follow-up semen analysis performed at 5 months. Baseline characteristics of the study population are as follows (Table 1): mean age was 34 years, mean age of partner was 33 years, and mean BMI was
28.7 with 18 individuals being overweight (BMI 25-30) and 12 individuals being obese $(\mathrm{BMI}>30)$. For patients with known smoking history, 25 of the 28 patients were non-smokers. In addition, $26.6 \%$ [8] of patients presented with at least grade 1 left varicocele, and $6 \%$ (2) of patients presented with at least a grade 1 right varicocele. 90\% [27] suffered from primary infertility and 10\% [3] suffered from secondary infertility.

In Table 2, baseline hormonal levels are compared with those attained at 1, 3 and 5 months. Focusing on the 5 -month hormonal profiles, there was a mean increase in testosterone (270.6 versus $412 \mathrm{ng} / \mathrm{dL}, \mathrm{P}<0.0001$ ), a mean decrease in estradiol (32 versus $15.9 \mathrm{pg} / \mathrm{mL}, \mathrm{P}=0.0059$ ), a mean increase in testosterone-to-estradiol (T/E) ratio ( 9 versus $26.5, \mathrm{P}<0.0001)$, a mean increase in the gonadotropin $\mathrm{LH}$ (3.4 versus $5.4 \mathrm{IU} / \mathrm{L}, \mathrm{P}<0.0001$ ), and a mean increase in the gonadotropin FSH (4.8 versus 7.5 IU/L, $\mathrm{P}<0.0001$ ). The aforementioned trends were statistically significant at 1 -month and 3-month follow-ups as well. No statistically significant difference was noted between baseline hematocrit and those measured at 1, 3 and 5 months.

The baseline semen parameters were also compared to semen parameters at 5 months. Significant increases were demonstrated in sperm concentration (7.8 versus 14.2 million $/ \mathrm{mL}, \mathrm{P}=0.0003$ ), total motile count (12.6 versus 17.7 million, $\mathrm{P}=0.0052$ ), and sperm morphology ( $3.0 \%$ vs. $3.5 \%, \mathrm{P}=0.0488)$. No significant changes from baseline were noted for semen volume and sperm motility (Table 3).

Overall pregnancy rate for our cohort was 46.6\% (14 of 30). Of the couples who achieved pregnancy, $71.4 \%$ (10 of 14) conceived through in vitro fertilization, $14.2 \%$ (2 of 
Table 2 Hormonal analysis of men treated with anastrozole

\begin{tabular}{|c|c|c|c|c|c|c|c|}
\hline Hormones & Baseline & Month 1 & $P$ & Month 3 & $\mathrm{P}$ & Month 5 & $\mathrm{P}$ \\
\hline Estradiol $(\mathrm{pg} / \mathrm{mL})^{\dagger}$ & $32.0(20.9-32.0)$ & $21.4(12.6-32.0)$ & 0.0085 & $17.8(15.0-32.0)$ & 0.0012 & $15.9(15.0-31.5)$ & 0.0059 \\
\hline T/E ratio ${ }^{\dagger}$ & $9.0(6.1-14.2)$ & $23.8(14.4-42.0)$ & $<0.0001$ & $23.1(14.5-33.7)$ & $<0.0001$ & $26.5(14.0-46.4)$ & $<0.0001$ \\
\hline $\mathrm{FSH}(\mathrm{IU} / \mathrm{L})^{\dagger}$ & $4.8(2.5-7.1)$ & - & - & $7.3(4.1-10.2)$ & $<0.001$ & $7.5(5.6-9.7)$ & $<0.0001$ \\
\hline
\end{tabular}

${ }^{\dagger}$ Median (IQR, interquartile range; 25th-75th percentile); ${ }^{\ddagger}$ Mean (SD, standard deviation); paired data differences: Month 1-Baseline, Month 3-Baseline, Month 5-Baseline were examined using 1-sample $t$-test or Wilcoxon signed rank test or Sign test. T/E, testosterone/estradiol; $\mathrm{FSH}$, follicle stimulating hormone; LH, luteinizing hormone.

Table 3 Semen parameters in subfertile males treated with anastrozole

\begin{tabular}{|c|c|c|c|c|c|}
\hline Semen parameter & Baseline & Month 5 & Actual change from baseline & $\%$ change from baseline & $P$ value \\
\hline Median (IQR) & $3.0(1.7-4.5)$ & $3.3(1.5-4.6)$ & $0.0(-0.5-0.8)$ & $0.0(-21.2-32.0)$ & $0.7670^{\dagger}$ \\
\hline \multicolumn{6}{|c|}{ Concentration (million/mL) } \\
\hline Median (IQR) & $7.8(2.6-19.6)$ & $14.2(5.5-34.0)$ & $5.2(0.7-14.8)$ & $52.8(30.0-275.0)$ & $0.0003^{\ddagger}$ \\
\hline Mean (SD) & $42.4(21.1)$ & $47.9(15.7)$ & $5.5(17.3)$ & $9.7(-12.7-117.4)$ & $0.0907^{\S}$ \\
\hline \multicolumn{6}{|c|}{ Total motile count (million) } \\
\hline Median (IQR) & $12.6(1.3-32.1)$ & $17.7(4.0-53.5)$ & $3.6(0.0-12.7)$ & $91.6(31.3-492.0)$ & $0.0052^{\ddagger}$ \\
\hline \multicolumn{6}{|c|}{ Strict morphology (\%) } \\
\hline
\end{tabular}

14) through intrauterine insemination and $14.2 \%$ ( 2 of 14) through natural intercourse. Anastrozole was well tolerated with mild side effects reported by only 3 patients out of $30: 1$ patient had minor headaches, 1 patient had mild malaise and 1 patient had elevated liver function tests.

\section{Discussion}

Spermatogenesis is a highly regulated process involving complex multifactorial hormonal regulation. The importance of intratesticular testosterone for male germ cell development is well established, but the complex effects of estrogen is still being elucidated. In general, the cumulative effects of estrogen are largely inhibitory; however, specific lower concentrations must be maintained to promote adequate spermatogenesis. The most understood inhibitory role of estrogens is on the hypothalamus and pituitary gland, leading to decreased gonadotropins ( $\mathrm{LH}$ and FSH). Estrogens also exhibit autocrine and paracrine effects on the testicular interstitium (Sertoli and Leydig cells). For instance, estrogens can modulate precursor populations of Leydig cells and reduce the stimulatory effects of $\mathrm{LH}$ on these cells causing decreased $\mathrm{T}$ production. Conversely, estrogens (along with FSH) promote spermatogenesis by aiding Sertoli cells in spermatogonia recognition and maturation. Hence, the role of estrogens in spermatogenesis is complex, but it is reasonable to conclude that high concentrations lead to decreased sperm quantity and quality (14-17).

The testosterone to estrogen ratio (T/E) provides a simple, quantitative comparison of these hormone 
concentrations. The T/E ratio was most notably used first by Pavlovich et al. to compare hormonal variations between infertile and fertile men (1). In this study, participants with severe male factor infertility had a mean T/E ratio of 6.9, while controls had a mean ratio of 14.5 . A cutoff of 10 as the lower limit of normal was subsequently established, which represented the 20th percentile of T/E ratios in that study. In our current investigation, we used this value to delineate normal versus abnormal $\mathrm{T} / \mathrm{E}$ ratios.

An abnormally low T/E ratio can be caused by decreasing testosterone, increasing estrogen, or more likely a combination of both processes. Hammoud et al. reported decreased sperm concentration in obese men to be associated with repeat polymorphism rate of TTTA nucleotides in the aromatase gene (11). Thus, one can infer that the overexpression of aromatase, a cytochrome P-450 enzyme that converts testosterone to estradiol and androstenedione to estrone, can lead to decreased $\mathrm{T}$ and increased $\mathrm{E}$. Aromatase is found throughout the body including the female reproductive tract, testis, liver, brain, and adipose tissue $(2,3)$. In the overweight (BMI $25-29.9 \mathrm{mg} / \mathrm{kg}^{2}$ ) and obese (BMI $\geq 30 \mathrm{mg} / \mathrm{kg}^{2}$ ) cohorts investigated in this study, the excess adipose tissue may contribute to the abnormal hormonal status, depressed semen parameters, and ultimately, their subfertility.

Anastrozole is a non-steroidal fourth generation aromatase inhibitor that was first approved in 1995 for the treatment of estrogen receptor positive breast cancer refractory to tamoxifen (18). Due to its mechanism of action that stops the conversion of testosterone to estradiol, anastrozole has also been used off label for the treatment of male infertility in males with an abnormal T/E ratio $<10$ in order to increase endogenous testosterone levels, with subsequent improvement in semen parameters [8-10]. Shoshany et al. further demonstrated that increased $\mathrm{T} / \mathrm{E}$ ratios significantly and directly correlated with improvement in semen parameters only in their cohort of 21 oligozoospermic men, treated with $1 \mathrm{mg}$ of oral anastrozole daily (8). In our study, we observed sustained increases in T/E ratios for up to 5 months of anastrozole therapy as well as improvement in semen parameters.

While the negative impact of elevated BMI on hormonal parameters has been consistently reported, the same cannot be said for semen parameters. The literature has shown various relationships between BMI and semen parameters, with some studies reporting no correlation, some studies reporting negative changes in specific individual parameters, and other studies finding depression in all semen parameters. In a systematic review and metaanalysis involving 115,158 total participants, Campbell et al. demonstrated that obese men were more likely to experience infertility, have decreased rates of live births per cycle with assisted reproductive technology (ART), and have a $10 \%$ absolute risk increase of pregnancy nonviability. In this study, significant differences were not found in conventional semen parameters, but obese men were found to have a higher percentage of sperm with low mitochondrial membrane potential (MMP), DNA fragmentation, and abnormal morphology (12). These findings suggest that while obesity may not affect traditional semen parameters, it may still promote infertility by inducing submicroscopic changes to sperm characteristics.

Our data corroborates much of the findings described in the literature regarding the effects of anastrozole on hormonal and semen parameters. More specifically, we found that in our cohort of overweight and obese men, anastrozole appropriately increased $\mathrm{T}$, decreased $\mathrm{E}$, and increased T/E ratio within 1 month of therapy and remained significantly different at 5 months of therapy. Sperm count, total motile count (TMC), and sperm morphology also showed statistically significant improvement, while semen volume and motility did not. Furthermore, fourteen of $30(46.6 \%)$ subjects achieved pregnancy, with majority conceiving through in vitro fertilization or through intrauterine insemination which suggests that anastrozole may be beneficial with aiding in conception for couples whose male partners are either overweight or obese.

One of the major limitations of this study was the use of $\mathrm{BMI}$ as an indicator of adipose tissue mass. Although BMI is widely used to determine an individual's weight status, it is often done so due to ease of calculation and may not correctly measure adipose content (2). In future studies, alternatives to BMI for body fat composition should be considered, such as the dual-energy X-ray absorptiometry (DXA) scan. Another limitation of this study was that the reproductive status of the female partners was unknown and could not be controlled for since our study was retrospective in nature. Although our findings achieved statistical significance with respect to semen parameters and hormonal profile, the power of our study was low and the efficacy of anastrozole for the treatment of the overweight, or obese subfertile male should be confirmed by a prospective, randomized clinical trial. Additionally, a future prospective, randomized clinical trial regarding response rates to aromatase inhibitors in men with normal 
BMI versus men with $\mathrm{BMI} \geq 25 \mathrm{~kg} / \mathrm{m}^{2}$ would be of great interest as well.

In summary, our study demonstrates improvement in hormonal parameters and semen parameters in subfertile men with BMI over 25. Overall, anastrozole is well tolerated with minimal side effects and may lead to improved pregnancy rates especially in conjunction with in vitro fertilization or intrauterine insemination. Therefore, we recommend anastrozole therapy for overweight or obese, subfertile males with abnormal T/E ratios to further aid in their attempts to achieve pregnancy naturally or with assisted reproductive techniques.

\section{Acknowledgments}

Funding: None.

\section{Footnote}

Reporting Checklist: The authors have completed the STROBE reporting checklist. Available at http://dx.doi. org/10.21037/tau-20-919

Peer Review File: Available at http://dx.doi.org/10.21037/ tau-20-919

Data Sharing Statement: Available at http://dx.doi. org/10.21037/tau-20-919

Conflicts of Interest: All authors have completed the ICMJE uniform disclosure form (available at http://dx.doi. org/10.21037/tau-20-919). The authors have no conflicts of interest to declare.

Ethical Statement: The authors are accountable for all aspects of the work in ensuring that questions related to the accuracy or integrity of any part of the work are appropriately investigated and resolved. The study was conformed to the provisions of the Declaration of Helsinki (as revised in 2013) and approved by the Ethics Committee of Hackensack University Medical Center/Institutional Review Board (IRB number: Pro00006190). Because of the retrospective nature of the research, the requirement for informed consent was waived. All patients' personal data was secure. The study outcomes will affect the future management of patients.

Open Access Statement: This is an Open Access article distributed in accordance with the Creative Commons Attribution-NonCommercial-NoDerivs 4.0 International License (CC BY-NC-ND 4.0), which permits the noncommercial replication and distribution of the article with the strict proviso that no changes or edits are made and the original work is properly cited (including links to both the formal publication through the relevant DOI and the license). See: https://creativecommons.org/licenses/by-nc-nd/4.0/.

\section{References}

1. Pavlovich CP, King P, Goldstein M, et al. Evidence of a treatable endocrinopathy in infertile men. J Urol 2001;165:837-41.

2. Inkster $\mathrm{S}$, Yue $\mathrm{W}$, Brodie $\mathrm{A}$. Human testicular aromatase: immunocytochemical and biochemical studies. J Clin Endocrinol Metab 1995;80:1941-7.

3. Schlegel PN. Aromatase inhibitors for male infertility. Fertil Steril 2012;98:1359-62.

4. Krausz C. Male infertility: pathogenesis and clinical diagnosis. Best Pract Res Clin Endocrinol Metab 2011;25:271-85.

5. Liu PY, Handelsman DJ. The present and future state of hormonal treatment for male infertility. Hum Reprod Update 2003;9:9-23.

6. Sussman EM, Chudnovsky A, Niederberger CS. Hormonal evaluation of the infertile male: has it evolved? Urol Clin North Am 2008;35:147-55.

7. Jarow JP, Zirkin BR. The androgen microenvironment of the human testis and hormonal control of spermatogenesis. Ann N Y Acad Sci 2005;1061:208-20.

8. Shoshany O, Abhyankar N, Mufarreh N, et al. Outcomes of anastrozole in oligozoospermic hypoandrogenic subfertile men. Fertil Steril 2017;107:589-94.

9. Raman JD, Schlegel PN. Aromatase inhibitors for male infertility. J Urol 2002;167:624-9.

10. Gregoriou O, Bakas P, Grigoriadis C, et al. Changes in hormonal profile and seminal parameters with use of aromatase inhibitors in management of infertile men with low testosterone to estradiol ratios. Fertil Steril 2012;98:48-51.

11. Hammoud AO, Gibson M, Peterson CM, et al. Impact of male obesity on infertility: a critical review of the current literature. Fertil Steril 2008;90:897-904.

12. Campbell JM, Lane M, Owens JA, et al. Paternal obesity negatively affects male fertility and assisted reproduction outcomes: a systematic review and meta-analysis. Reprod Biomed Online 2015;31:593-604. 
13. Alshahrani S, Ahmed AF, Gabr AH, et al. The impact of body mass index on semen parameters in infertile men. Andrologia 2016;48:1125-9.

14. Schulster M, Bernie AM, Ramasamy R. The role of estradiol in male reproductive function. Asian $\mathrm{J}$ Androl 2016;18:435-40.

15. Van Beurden WM, Roodnat B, Mulder E, et al. Further characterization of the effects of hypophysectomy, FSH and estrogen on LH stimulation of testosterone production in Leydig cells isolated from immature rats. Steroids 1978;31:83-98.

16. MacCalman CD, Getsios S, Farookhi R, et al. Estrogens potentiate the stimulatory effects of follicle-stimulating hormone on $\mathrm{N}$-cadherin messenger ribonucleic acid levels in cultured mouse Sertoli cells. Endocrinology 1997;138:41-8.

17. MacCalman CD, Farookhi R, Blaschuk OW. Estradiol regulates $\mathrm{E}$-cadherin $\mathrm{mRNA}$ levels in the surface epithelium of the mouse ovary. Clin Exp Metastasis 1994;12:276-82.

18. Wiseman LR, Adkins JC. Anastrozole. A review of its use in the management of postmenopausal women with advanced breast cancer. Drugs Aging 1998;13:321-32.

Cite this article as: Shah T, Nyirenda T, Shin D. Efficacy of anastrozole in the treatment of hypogonadal, subfertile men with body mass index $\geq 25 \mathrm{~kg} / \mathrm{m}^{2}$. Transl Androl Urol 2021;10(3):1222-1228. doi: 10.21037/tau-20-919 